\title{
Very Slow Exchange of Sulfur Atom between Sulfate and Dodecyl Sulfate lons
}

\author{
Mitsuo Muramatsu and Kyoko NaKao* \\ Department of Chemistry, Faculty of Science, Tokyo Metropolitan \\ University, Setagaya-Ku, Tokyo
}

Received July 24, 1967

\section{Introduction}

Radioactive sodium dodecyl sulfate (SDS) has been used for studying various surfacechemical processes such as adsorption at solution surfaces ${ }^{1)}$, incorporation into fabric $\operatorname{cotton}^{2)}$, and self-diffusion in aqueous media ${ }^{3)}$, using solutions of SDS alone or SDS accompanying various salts such as sodium or potassium sulfate which is currently used as a builder in detergency. In many cases of these experiments, SDS is labeled with ${ }^{35} \mathrm{~S}$ under the assumption that the S-containing group in the molecule remains unchanged throughout the processes. To verify the assumption, one has to confirm the slowness to the hydrolysis reaction,

$$
\begin{array}{r}
\mathrm{C}_{12} \mathrm{H}_{25} \cdot \mathrm{O} \cdot{ }^{35} \mathrm{SO}_{3}{ }^{-}+\mathrm{H}_{2} \mathrm{O} \rightarrow \\
\mathrm{C}_{12} \mathrm{H}_{25} \mathrm{OH}+\mathrm{HO} \cdot{ }^{35} \mathrm{SO}_{3}{ }^{-},
\end{array}
$$

in comparison with the surface-chemical processes involved in SDS-water system. We thus measured the radioactivity of ${ }^{35} \mathrm{SO}_{4}^{2-}$ formed by the reaction (1), for which ca. 40 years and 180 days, for instance, were found as half life for the solutions of $\mathrm{pH} 4$ and 1 , respectively, and of initial concentration of $1 \times 10^{-3} \mathrm{~mol} / /^{4}$.

If, however, the system contains inorganic sulfate(s) added to SDS-water system, one must take an additional account of the exchange reaction,

$$
\begin{aligned}
& \mathrm{C}_{12} \mathrm{H}_{25} \cdot \mathrm{O} \cdot{ }^{35} \mathrm{SO}_{3}^{-}+\mathrm{O} \cdot \mathrm{SO}_{3}^{2-} \rightleftarrows \\
& \mathrm{C}_{12} \mathrm{H}_{25} \cdot \mathrm{O} \cdot \mathrm{SO}_{3}^{-}+\mathrm{O} \cdot{ }^{35} \mathrm{SO}_{3}^{2-} ;
\end{aligned}
$$

otherwise, confusion may take place with the radioactivity of $\mathrm{SDS}^{35} \mathrm{~S}$ as an expression of the process involved.

Present paper deals with the exchange reaction (2) in relation to the hydrolysis (1), as well as to the similar exchange reactions between sulfate and sulfonates. It has been reported that the sulfur atom of inorganic sulfate does not exchange appreciably with that of various organic sulfonates such as benzene sulfonate and its derivatives ${ }^{5}$.

\section{Experimental}

Sodium sulfate labeled with ${ }^{35} \mathrm{~S}$ (specific activity, 5-6 $\mathrm{Ci} / \mathrm{mol}$ ) and non-radioactive SDS were mixed to form aqueous solutions of concentration ranging (1-4) $: 10^{-3} \mathrm{~mol} / \mathrm{l}$ for each component. The acidity was kept to ca. pH 1 for all solutions, as acidic medium was found to enhance the reaction $(1)^{4)}$. The exchange mixture was kept standing in a thermostat at $40-60^{\circ} \mathrm{C}$. After a given time, an aliquot of $0.5 \mathrm{~m} l$ of the mixture was taken out to make $100 \mathrm{~m} l$ of precipitation mixture which contained additional $6 \times 10^{-5} \mathrm{~mol}$ of non-radioactive SDS as a carrier. Immediately, $3 \times 10^{-5} \mathrm{~mol}$ of barium chloride of as high concentration as possible was added to the precipitation mixture. Barium dodecyl sulfate (BDS) thus formed was filtered, washed and mounted for counting radioactivity by Aloka FC-IE $2 \pi$ windowless gas flow counter.

Sodium sulfate used was purified by repeated recrystallizations. SDS was synthesized by

* Present address: Research Institute of Tuberculosis, Kiyose-Machi, Tokyo 
the reaction of dodecanol with sulfonic acid and purified thoroughly to give no depressions in surface tension-concentration curve.

\section{Results and Discussions}

It is always a problem how to separate an exchange species from the precipitation mixture with least contamination. In the present experiments, the separated BDS was accompanied more or less by sulfate whose specific activity was much higher than that of BDS. Fig. 1 gives the radioactivity of $\operatorname{BDS}$ as a function of $\mathrm{pH}$ of the precipitation mixture at exchange time to be zero, viz., when the precipitation procedures followed in the wake of formation of the exchange mixture. It can be seen in Fig. 1 that the co-precipitation of sulfate with BDS is minimized at around $\mathrm{pH} 11$, where radioactivity of $20-60 \mathrm{cpm}$ was always found in BDS sample obtained. We have thus taken this $\mathrm{pH}$ value for acidity of precipitation mixture.

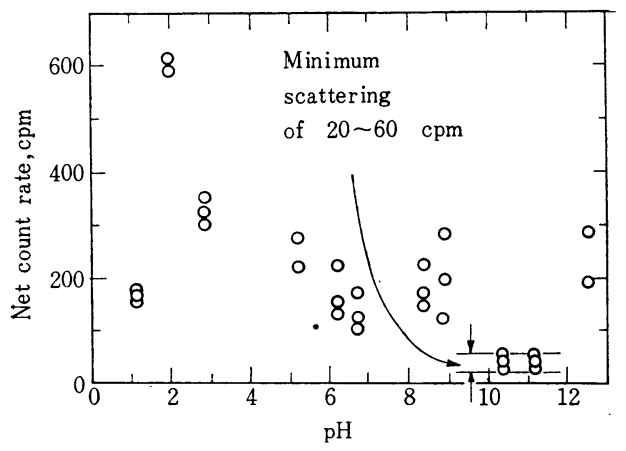

Fig. 1 Effect of $\mathrm{pH}$ of precipitation mixture upon radioactivity of inorganic sulfate accompanying BDS.

Precipitation mixture contains $5 \times 10^{-7}$ mol of sodium sulfate and $5 \times 10^{-7} \mathrm{~mol}$ (original) plus $6 \times 10^{-5} \mathrm{~mol}$ (carrier) of SDS. Exchange time, $0 \mathrm{hr}$; Temperature, $60^{\circ} \mathrm{C}$.

It was found that the radioactivity of BDS was not increased with elapsing the time of exchange reaction. This was indeed the case with any combination of concentrations of SDS and sulfate, so far tested, and at any temperature up to $60^{\circ} \mathrm{C}$. Typical example is shown in Fig. 2 in which the count rate is plotted against the elapsed time. As is usuals of this sort of experiment, the count rate gives a fluctuation due to the irregular coprecipitation. It should be recognized, however, that the fluctuation is almost within the inevitable scattering of $20-60 \mathrm{cpm}$ given in Fig. 1. It must also be mentioned that the hydrolytic reaction (1) using SDS- ${ }^{35} \mathrm{~S}$ of same specific activity has resulted in an increment of $10^{4}-10^{5} \mathrm{cpm}$ at the elapsed time of 14 days under the similar expeimental conditions $\mathrm{s}^{4}$. Therefore, exchange rate of the reaction (2), if any, should be of the order of magnitude of at least $10^{5}-10^{6}$ years in its half life. Such a slowness of the reaction (2) seems to permit us to use a system containing SDS- ${ }^{35} \mathbf{S}$ and $\mathrm{SO}_{4}^{2-}$ without care about the ambiguity as to the radioactivity measured thereof.

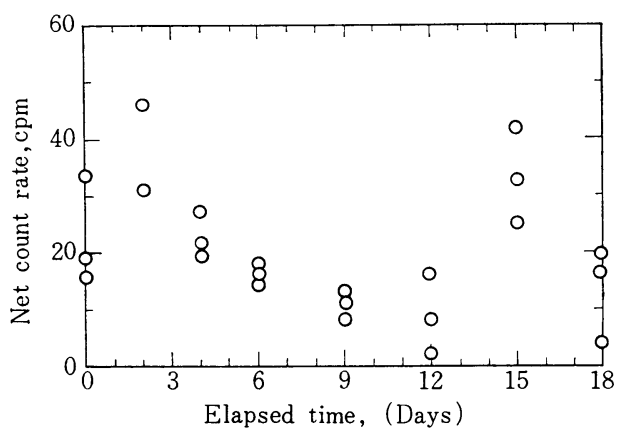

Fig. 2 Effect of elapsed time upon radioactivity of $\mathrm{BDS}$.

Exchange mixture, $1.0 \times 10^{-3} \mathrm{~mol} / l$ for each of SDS and sodium sulfate; $\mathrm{pH}$ 1. 13; Temperature, $40^{\circ} \mathrm{C}$.

It has been a problem whether $\mathrm{C}_{12} \mathrm{H}_{25}-\mathrm{O}$. $\mathrm{SO}_{3}^{-}$or $\mathrm{C}_{12} \mathrm{H}_{25} \cdot \mathrm{O}-\mathrm{SO}_{3}^{-}$bond rupture is involved in the hydrolytic reaction $(1)^{4}$. It seems that the present results suggest the likelihood of the latter possibility rather than the former which should lead to easier exchangeability with sulfate ion. This suggestion is in accord with the conclusion that the hydrolytic reaction of carboxylate ester involves the rupture of $\mathbf{R} \cdot \mathbf{O}-\mathrm{CO} \cdot \mathbf{R}^{6}$.

The authors thank Professor Sasaki for his interest in this work. The cost of this research has been defrayed by the Scientific Research Expenditure Grant of the Ministry of Education given to him. 


\section{References}

1) Salley, D.J., Weith, A.J., Jr., Argyle, A. A., and Dixon, J.K.: Proc. Roy. Soc. (London), A203, 42 (1950)

2) Fava, A., and Eyring, H.: J. Phys. Chem., 60, 890 (1956)

3) Matuura, R.: Kagaku to Kogyo(Chemistry and Chemical Industry), 10, 997 (1957)
4) Muramatsu, M., Inoue, M., and Sasaki, T.: "Proc. 7th Japan Conf. on Radioisotopes", Japan Atomic Energy Ind. Forum, Tokyo (1966) p. 240

5) Muramatsu, M.: Bull. Chem. Soc. Japan, 32, 290 (1959)

6) Polanyi, M., and Szabo, A. L.: Trans. Faraday Soc., 30, 508 (1934) 